\title{
Industry views on water resources planning methods- prospects for change in England and Wales
}

\author{
Sean Turner ${ }^{1}$ and Paul Jeffrey ${ }^{2}$
}

\begin{abstract}
This paper describes a qualitative study of practitioner perspectives on regulated water resources planning practice in England and Wales. The study focuses on strengths and weaknesses of existing practice and the case for change toward a risk-based approach informed by stochastic modelling assessments. In-depth, structured interviews were conducted to capture the views of planners, regulators and consultants closely involved in the planning process. We found broad agreement that the existing water availability assessment methods are fallible; they lack transparency, are often highly subjective, and may fail to adequately expose problems of resilience. Whilst most practitioners believe these issues warrant a more detailed examination of risk in the planning process, few believe there is a strong case for a fundamental shift toward risk-based planning informed by stochastic modelling assessments. The study identifies perceived business risks associated with change and exposes widespread scepticism of stochastic methods.
\end{abstract}

Key words. Water resources; water supply; water supply and demand; water industry; simulation modelling; risk assessment; modelling; uncertainty analysis.

\section{Introduction}

Water resources planning practice in England and Wales has undergone significant change over the last 25 years. It has responded to the political, legislative and regulatory environment to become a formal, standardised process. It has amalgamated old and new concepts, and crystallised them in a consistent and clearly defined set of metrics and methods that form the building blocks of the Water Resources Management Plan (WRMP) — now a statutory reporting requirement for all water companies. Yet none of the methodological changes experienced since industry privatisation represent a fundamental shift away from classic design principles that have persisted for more than a century. Ever present features include: an assessment of water availability under a given set of conditions and assuming historical recorded inflows, which produces a deterministic metric of supply known internationally as "system yield" and today in England and Wales as "Deployable Output" (DO); the use of subjectively defined planning heuristics and engineering margins; and the communication of

\footnotetext{
${ }^{1}$ Corresponding author. Singapore University of Technology and Design, 20 Dover Drive, Singapore 138682, Email: sean_turner@sutd.edu.sg

${ }^{2}$ Cranfield Water Science Institute, Cranfield University, Bedford, UK. MK43 OAL. 
the state of the system using deterministic terminology, such as "surplus" and "deficit", which can also be quantified as the difference between yield and demand - the so-called "supply-demand balance."

Under these design principles, an impetus for investment is created if current or projected conditions open up a deficit between supply and demand. The aim is then to discover the least-cost combination of options to address the deficit over the planning horizon. This design paradigm is broadly known as "least cost capacity expansion" (Loucks et al., 2005). Its weaknesses from a technical decision making perspective, which have long been recognised and are well documented, derive from its deterministic outlook amidst hydrological uncertainty and other uncertainties, which limits prospects for weighing the costs of infrastructure development against the benefits in terms of reduced risk. Capacity expansion planning contrasts with "risk-based" forms of planning that integrate supply and demand in the modelling assessments, explore hydrological variability and uncertainty using stochastically-derived flows and extensive Monte-Carlo analysis of the system, and produce likelihood estimates for a range of undesirable drought-related hazards.

A debate on whether to shift toward this form of planning has begun in England and Wales, where a number of recent academic papers have promoted new planning frameworks that purport to deal more effectively with uncertainty (e.g., Hall et al., 2012; Hall and Borgomeo, 2013; Korteling et al., 2013; Matrosov et al., 2013). More generally, there is a view from academia and consulting firms that the climate change adaptation agenda demands a fresh approach for dealing with uncertainty in water resources planning practice (views elicited by CH2MHill on behalf of the Environment Agency, 2013). This contrasts with the more conservative ambitions of the water companies (same study), which, in general, seek changes that would simplify and clarify existing process. So far this discussion has suffered from a lack of well-evidenced, documented knowledge to explain why the opinions of academics and practitioners diverge so starkly. This study aims to address this knowledge gap by capturing practitioner perspectives on the role of modelling and analysis in water resources planning. It seeks to understand what practitioners want to achieve through their modelling assessments and to expose the relevant viewpoints to help guide future research and inform policy decisions on how to shape the WRMP guidelines for 2019 and beyond.

\section{Interview Approach}

The study targeted experienced practitioners with working knowledge of the water availability assessment methods prescribed by the WRMP guidelines (EA, 2012). Our focus on technical methodological aspects distinguishes the study from earlier research (e.g., Davies and Daykin, 2011) that elicited practitioner views on the more general and administrative aspects of the WRMP process. The study is also distinct from the "Manual of Source Yields" UK Water Industry Research (UKWIR) project and the aforementioned work examining the case for change in the planning guidelines (EA, 2013) because it seeks to 
explicate some of the underlying motives that shape practitioner opinions on planning methodology.

The research sought to answer three questions:

1. How do some of the accepted technical methodological weaknesses of DO assessments affect the ability of companies to plan effectively?

2. Why might practitioners seek to uphold the conventional modes of planning?

3. What institutional factors might hinder the prospects for a risk-based approach informed by stochastic modelling assessments?

We interviewed fifteen practitioners across seven water companies, the Environment Agency, Ofwat and two consulting firms. The sampling of participants was non-random; practitioners were selected for interview based on their role, experience and level of involvement with recent regulated planning activities. A small number of participants were recommended by other interviewees as appropriately experienced to contribute to the study. The sample incorporated a mix of lead planners (e.g., Head of Water Resources, Supply-Demand Manager), modellers (e.g., Modelling Manager, Water Resources Analyst/Consultant) and regulators (e.g., national-level co-ordinator, Regional Officer). All prospective interviewees were approached via email.

The interviews were conducted face-to-face, which allowed for the use of visual aids and sketches for explaining and discussing abstract ideas. The interviews were "in-depth," comprising pre-determined open-ended questions (i.e., "Why...?" and "How...?" questions rather than questions that can be answered with yes or no) and impromptu follow-up questions designed to extract as much information as possible on each subject (Foddy, 1993). A structured script was followed to prevent the discussion from drifting and to allow for responses to be categorised and compared. We used hypothetical scenarios-presented using water resources system schematics and performance graphs - to develop shared understanding and ensure questions were understood as intended. The script also included prompt information, including quotations from industry reports and reasoned arguments; the intention was not to lead the participant but rather to evoke counter-arguments and opinions on contentious issues, specifically those relating to weaknesses in DO assessment methodology (the approach was to first establish whether the interviewee acknowledged the existence of a particular weakness and then to elicit views on how the issue might affect a company's ability to plan effectively). A basic interview script was sent to participants in advance to give them the opportunity to understand the interview themes and raise any questions. All participants were advised that their answers would be reported in anonymous form, with the proviso that distinctions might be drawn between different groups of interviewed practitioners (i.e., regulators versus company planners). The typical interview lasted between 60 and 90 minutes.

Results are reported in the following sections using non-quantified terms - one, a few, some, most, almost all, all, etc. - to deter the reader from inferring proportional industry-wide 
representation from the relatively small sample of participants. All participants are considered to be 'practitioners.' Company practitioners are termed 'planners.' Practitioners from the Environment Agency and Ofwat are termed 'regulators.' Practitioners from consulting firms are termed 'consultants.'

\section{Practitioner Perspectives}

\section{Establishing weaknesses in DO assessment methodology}

Three separate hypothetical scenarios were presented to elicit views on some possible weaknesses in DO assessment methodology. The scenarios were fabricated, but had been set up using real reservoir inflow data and modelled using Aquator (Oxscisoft). The first scenario was designed to highlight a problem of capturing resilience using DO assessments. It featured two hypothetical single-reservoir Water Resource Zones (WRZs): one was in a state of DO deficit, but was supported by a desalination plant that would be able to supply a third of the demand if the reservoir failed; the other was in DO surplus, but was isolated such the zone would suffer total demand shortfall if the reservoir failed (i.e., the zone in deficit was more resilient than the zone in surplus). Participants were presented with supply-demand balances and supporting risk analyses describing the state of both systems. They were then asked in which of the two systems they would invest an arbitrary sum of money for alleviating risk, ignoring regulatory guidance (which would direct investment to address the DO deficit) and assuming only one system could be selected for improvement. Results are presented in Box 1.Very few participants - and no company planners - argued that the system in deficit merited the investment. Roughly half of the participants reported that the system in surplus merited the investment, in most cases commenting that the consequences of failure in that system would be unacceptable. Most of the other participants reported that they would be uncomfortable reaching a decision without further information, particularly customer preferences.

\section{Box 1}

In which system would you invest - the system in deficit (incorporating desalination support) or the system in surplus (but isolated)?

System in deficit $\quad$ System in surplus $\quad$ Unwilling to commit
$\sim$ Half of the participants
Very few
Almost half

The second scenario focused on the issue of subjectivity. The scenario showed how a reservoir system could shift from a state of significant DO surplus to significant DO deficit depending on the size of the Emergency Storage, which is influential if DO is constrained by critical drought failure. Participants were asked for their interpretation of the reasons behind industry rules for sizing and reporting of the Emergency Storage. Most participants commented that the Emergency Storage margin came out of the Government's Agenda for 
Action (DoE, 1996) that followed the 1995/96 drought, but none knew why 30 days' demand is the recommended volume. Some participants guessed that the assumption had been based on an assessment of the time required to either ensure supply into wetter autumn months or to implement contingency measures. Others believed the reasoning was more superficial: 30 days is a "nice number," equal to a calendar month. But most either gave no reason or stated explicitly that there is no logical reasoning behind the volume used. A few participants reported that Emergency Storage is too difficult an issue to tackle properly and, as such, it has never been challenged in a risk management context. Moreover, the majority of participants believed that the omission of Emergency Storage in guidelines and reporting requirements is an oversight - that the Emergency Storage "has slipped through the net", is "lost in history", and has been "buried in the analysis and forgotten about." One planner described a "tick-box mentality" that has led regulators to overlook important details, including Emergency Storage. A few participants commented that such subjective assumptions lead to a weak understanding and prevent clear communication of risk to customers.

A third scenario focused on comparability of DO assessments. The aim was to highlight how certain system characteristics (e.g., level of integration and source diversity) might prevent fair comparison of DO assessments across different zones. The scenario turned out to be superfluous because the idea that DO assessments cannot be fairly cross-compared across WRZs was already an accepted fact in nearly all of the participants' minds. Most reported that fair DO cross-comparison was an unrealistic goal and that there are numerous causes of inconsistency across different WRZs, including emergency storage, source types and control curve positioning.

\section{Implications for effective planning}

The basic scenarios and subsequent discussions established wide practitioner agreement that DO assessments (1) may fail to capture problems of resilience, (2) are often influenced by highly subjective assumptions, and (3) cannot generally be compared across different WRZs. Unsurprisingly then, nearly all of the practitioners reported that there was a case for a more detailed exploration of risks in the guideline methodology (Box 2). Several participants suggested there was a need to extend the understanding of resilience and the consequences of system failure. Others argued that there was a need to better communicate the risks, costs and trade-offs in the plans. One participant reported that there was a need to recognise the value of investments that extend supply-demand surplus. Moreover, several participants added to the critique of existing DO assessment methodology by identifying some additional problems, including: a lack of any requirement to estimate likelihoods for a repeat of the drought of record or for a drought that would cause catastrophic failure; a failure to adequately expose the risk trade-offs between different interest groups; a lack of any requirement to understand and expose the "real consequences" of drought (e.g., would standpipes really be implemented at the lower storage triggers, or would a state of civil emergency generate sufficient political impetus to begin breaching environmental thresholds to uphold customer supplies?); and a failure to capture "robustness" - the capability of a system to uphold required performance standards under many alternative plausible futures. 


\section{Box 2}

Is there a case for a more detailed exploration of risk for informing water resources planning decisions?

\section{$\underline{Y e s}$}

Nearly all participants $\underline{\text { No }}$

Very few $\underline{\text { Unsure }}$

Very few

Yet despite all of the issues identified above, most of the participants argued that the existing process is imperfect but adequate; only a small minority saw any need for fundamental changes in the planning process. The identified weaknesses (resilience, subjectivity, inconsistency, etc.) were not widely regarded as a major impediment to effective planning within the current framework. The general line of thinking here was that DO assessment, despite its fallibilities, does not necessarily constrict a company from accommodating other sources of information in its plan; the overall process allows flexibility for important elements to be incorporated even if DO assessment fails to expose them. This view was typified by one participant's claim that existing process is not "black and white"-it can accommodate political influence, the experience and knowledge of planners, or even additional analysis. One practitioner cited published planning appendices to demonstrate that certain companies had investigated resilience separately as part of their plans. On the issue of subjective margins, many participants emphasised that the aim of the prescribed planning process is not to attempt to define the "right" level of protection, but to define a baseline against which alternative options and scenarios can be tested. These practitioners argued that the primary function of the planning guideline is to provide transparent and consistent principles, minimum technical standards, an easily-understood basis for asking questions and a systematic, simple and repeatable method. Few saw any need for the baseline DO assessments to be comparable across the different water companies (although nearly all believed that a company should be able to cross-compare the water availability assessments across its own WRZs). The general feeling was that consistency of method and principles was much more important than comparability of the output metrics.

Some participants also discussed ways in which to improve current practice without shifting away from DO assessment and capacity expansion planning. For example, a few participants suggested that the Emergency Storage margin could be removed and the underlying uncertainty brought into Headroom to remove some of the subjectivity and improve transparency.

\section{Effective communication}

Participants were asked what possible problems might arise from communicating risk in a simplistic way through the supply-demand balance (the question was primed with a statement noting that customers may assume that "surplus" indicates zero likelihood of failure, or that "deficit" indicates extreme risk). A few practitioners reported that stakeholders can be easily 
misled by the supply-demand balance - it "hides complexity," "creates a blinkered view of what's going on" and creates a false impression that "right level of risk is known." One participant reported that politicians tend to get an oversimplified impression that a certain level of investment can eradicate risk, which fosters distrust in the industry if and when the fragility of a system is exposed by severe drought conditions. Another noted that the supplydemand balance fails to present the cost-benefit trade-off. One participant suggested that term "surplus" might create a misleading impression that water that can be traded away without significant impact on risk.

Interestingly, most practitioners spontaneously attempted to justify the need to communicate the state of a WRZ using a supply-demand balance. Many argued that the supply-demand balance is the only way to get an informed response from customers. The dominant belief was that companies need a simple and "clear" way of alerting customers to any issues, and that the best way to achieve this is with a pass/fail test and the term "deficit." This perspective re-emerged at a number of different points during the interview. For example, when asked why classic design principles have persisted, a number of practitioners focused on the need for a clear pass/fail test, because "customers will tend to agree that a deficit needs to be addressed." One practitioner captured the general sentiment by reporting that the supply-demand balance is more important as a communication tool than as a means to reaching decisions on where and how to invest in system improvements. Similarly, when asked about the overall purpose of the planning process, a number of participants focused on the value of prescribed guidance in terms of the legitimacy it creates for helping build the case for investment. They reported that the main purpose of the guideline is to expose risks, to identify and explicate the need for new resources and, ultimately, to "help companies invest to protect supplies for customers."

\section{Prospects for risk-based planning}

It appears that an important advantage of DO assessment is its deterministic output, which helps planners communicate risk in simple terms. It should follow then that a method that produces complex metrics that are difficult to communicate would be deemed unattractive. Indeed, the interviews confirmed this supposition. Several participants reported that stakeholders would struggle to understand probabilistic performance metrics. Some feared that exposing difficult risk-cost trade-offs might open up non-productive debate on whether and how to invest. One participant used the term "paralysis by analysis" to describe this risk; another stated that investment based on probabilities would be a "hard sell." One participant also warned that stochastic hydrology would cause confusion and emphasised the importance of using DO based on the drought of record, which provides clear assurance to customers that the system is designed against a real and tangible event.

Several participants questioned the validity, rigor and worth of risk-based planning frameworks from a decision making perspective. For example, a third of participants indicated distrust in the plausibility of synthetic droughts generated by stochastic models and suggested that sensitivity analysis of the drought of record would be a more rigorous and 
appropriate way to examine risk and uncertainty. Some practitioners also identified potential problems with defining the "right level of risk" and others commented that companies would struggle to adequately monetise risks for project appraisal. A small number of participants challenged the idea that existing practice fails to accommodate risk-they argued that the Target Headroom percentile provides adequate scope for effective risk-cost trade-off.

In contrast to these views, several practitioners reported that a risk-based planning framework would provide additional rigour and a stronger foundation for decision making, but that the industry lacks the time, resources and technical capacity to undertake the extensive modelling assessments that underpin this approach. Some practitioners also identified business and regulatory risks associated with overhauling the planning methodology. For example, certain companies might be uncomfortable informing stakeholders that the previous analysis was "wrong" in the event that a new form of analysis recommends a radically different course of action. A number of company planners indicated that regulators are resistant to major change because they have invested a lot in current approach, which is "tried and tested." Some participants reported that the industry was collectively aware that existing practice is fallible, but that there has been a lack of demonstration of alternative approaches.

Box 3 summarises the reasons why the water industry in England and Wales might resist a fundamental change in planning practice toward a risk-based approach.

Box 3 Reasons for resistance to fundamental change toward a risk-based approach

Too hard to communicate probabilistic metrics to stakeholders

Existing process is flexible enough to accommodate risk

Methods necessary to support a risk-based analysis lack rigour

Methods necessary to support a risk-based analysis are too challenging, time consuming and/or expensive for companies to undertake

A viable alternative to existing practice not yet demonstrated

Planning process is less about finding the right level of risk than it is about providing a systematic basis for asking questions

Planning process is less about finding the right level of risk than it is about legitimising decisions that might be reached via means other than technical assessments (e.g., planner experience, political influence)

Change would risk confusing and losing the trust of stakeholders

Too much invested in existing process to change (most participants)

(several)

(several)

(afew)

(afew)

(afew)

(a few)

(very few)

(very few) 


\section{Discussion}

\section{The role of modelling assessments in planning}

The interviews exposed an interesting tension relating to the adequacy and effectiveness of DO assessment (and corresponding supply-demand balance) for communicating the state of a WRZ. To illustrate, no participant was able to provide a logical line of reasoning for the use of a specific margin of Emergency Storage; many even discussed how this feature has been buried in the analysis and forgotten about. Yet many of the participants also lauded the supply-demand balance on the basis of "clear communication" of the state of the WRZ. This raises an intriguing question: how can the measure of supply be deemed "clear" if it relies on such a significant level of "buried" subjectivity? And why did some participants report that the supply-demand balance provides a "clear" form of communication whilst others reported the exact opposite? One suspects the answer lies in the use language rather than in a divergence in opinion. "Clear" is probably the wrong word; a more accurate term might be "screened." A deterministic measure of supply conceals complexity and thereby mitigates difficult questions. Few people would argue that "supply" should not meet demand or that a "deficit" should not be addressed, primarily because the information is presented in such a way that encourages the viewer to ignore the fact that "supply" is a complex and indeterministic concept. Conversely, there is plenty of scope for argument on whether a water company should aim for $75 \%$ or $95 \%$ certainty that it will maintain a restriction Level of Service of 1 in 20 years. Or should it aim for $95 \%$ certainty that the 1000-year drought will not cause catastrophic failure? Why not the 1 in 1500-year drought — and 98\% certainty - just to be on the safe side? Would that be worth the $£ 100$ million investment? The scope for debate is endless, which is why one participant used the term "paralysis by analysis" to describe the risk of stifling a company's ability to build consensus and legitimise a course of action.

Indeed, most participants made a similar point in a less forthright way by suggesting that stakeholders would struggle to understand probabilistic performance metrics, or that presenting a supply-demand balance is the "only way to get an informed response." This angle is slightly (and probably unintentionally) disingenuous because it assumes that stakeholders understand, and are adequately informed by, a supply-demand balance. The reality is that the only person that understands a supply-demand balance is the person who makes the underlying assumptions necessary to define supply in a deterministic way. Even if outsiders rigorously inspect the publically available planning documents they may still fail to gain such understanding, as several companies either fail to report on particular assumptions or withhold technical documents that outline those assumptions. The point is not to question or castigate the motives of planners, but simply to highlight the paradoxical nature of statements that emphasise the need for "clear" communication through a deterministic metric of supply. Taking these statements for what they really mean, the need to build consensus and generate an impetus for action through the modelling output is evidently important to a large section of the planning community. If a move away from deterministic planning prevents or 
hinders a company's ability to invest in water resources infrastructure, then it also creates significant business and regulatory risk. Such risks have been largely ignored in the academic literature promoting fundamental change in the planning process and perhaps deserve more attention in future academic discussions on how to shape the planning process for WRMP19 and beyond.

\section{Implications for existing practice}

It appears that effective decision making and effective project implementation can be conflicting objectives. On one hand, planners seek to use modelling assessments to develop a clear picture of risk and to understand how different options might address areas of concern.

Nearly all of the participants agreed that there is a case for more detailed examination of risks in the planning guideline in order to help achieve those aims. On the other hand, a large section of the planning community appears reluctant to communicate the state of their WRZs in uncertain terms; the modelling assessment must legitimise action as well as inform it. The interview responses provide some insights to inform a discussion of the potential issues associated with accommodating these conflicting goals in a deterministic planning framework.

The use of DO analysis and a supply-demand balance does not necessarily preclude more complex forms of decision making. On the contrary, many participants described additional components of decision making that can take place outside the DO assessment procedure. Knowledge of experienced practitioners, political considerations and separate analyses (e.g., vulnerability/resilience analysis) were cited as important sources of information that inform the ultimate investment decisions. Of course, accommodating these aspects will present a challenge in cases where perceived investment needs fail to match with the outputs of the prescribed modelling procedure. How will a company with a resilience problem, for instance, invest to deal with that issue if its WRZ is in a state of surplus? One can only assume that participants referring to the "flexibility" of existing practice meant that modelling assumptions can be adjusted in order to legitimise the desired course of action. This form of behaviour may seem illicit, but there are few alternatives for reconciling strict adherence to a modelling procedure for justifying decisions with the need to allow for important factors that cannot be easily captured through those modelling assessments.

Several participants acknowledged this issue and reported that the prescribed process primarily aims to set a consistent basis for asking questions - that is, companies are not strictly bound by the least-cost capacity expansion ethos and may reasonably deviate from it by, for instance, favouring a set of options that do not necessarily resemble "least cost" for addressing a supply-demand balance but provide some other benefit (such as dealing with resilience). Challenging this view, one participant reported that companies that have faced public inquiry for their WRMPs had suffered extensive scrutiny focused specifically on the parts of their plans that deviated from the prescribed process. Moreover, there are other regulatory reporting requirements, such as the Security of Supply Index (SOSI), that derive from the supply-demand balance, so one cannot assume that companies would always be able 
to deviate from it without implications. So whilst in theory a company may be able to accommodate alternative aspects in the existing process in a clear and transparent way, in practice there are business risks associated with this approach. Regulators should perhaps consider that the current setup and the potentially conflicting objectives of decision-making and project justification may incentivise manipulation of modelling assumptions.

There may be wider risks associated with the use of a supply-demand balance for communicating the state of a WRZ, particularly where those interpreting it are unaware of its limitations. Some participants considered this issue, envisioning potential problems where politicians get the wrong impression. For example, it is well acknowledged among the practitioner community that three consecutive dry winters is plausible hazard and a significant risk for southeast England. Yet faced with a supply-demand balance in a state of surplus, politicians may overlook this threat. Similarly, people outside the water resources planning community are unlikely to be aware that DO assessments cannot be fairly crosscompared across different companies. For example, The UK Infrastructure Transitions Research Consortium recently published a national assessment of water security using aggregated DO data (UK ITRC, 2014). If a consortium of infrastructure planning experts based at the country's most prestigious academic institutions failed to recognise what is concealed by the current planning metrics then what can be expected of Government policy makers and others viewing these assessments? As one participant noted, these issues of comparability could become increasingly important if and when companies begin to consider water trading.

\section{Limitations and future research needs}

The interview study captured the views of only fifteen practitioners. Moreover, the sample was confined to planners, consultants and regulators. Therefore the identified business risks may fail to adequately represent wider industry and company opinion. Company directors, for example, might have expressed a different set of views relating to the role of modelling assessments in building a consensus for action. Moreover, since the prospect of stochastic modelling is nascent in England and Wales, the scope for detailed discussion on emerging planning methods was somewhat restricted. There was considerable variation in the participants' level of understanding of what stochastic modelling assessments might achieve. The industry may offer a more balanced and considered perspective as the new methods find their way into UKWIR projects and national WRMP meeting agendas. A more detailed and expansive interview study would perhaps be of greater value in two or three years' time as industry regulators begin to set down the WRMP guidelines for 2019. A broader range of scenario types, including examples featuring groundwater-only systems (of which there are many in England and Wales), would both enhance the robustness of the study and help widen its scope to better accommodate the views of a large and important section of the practitioner community.

The interviews exposed a number of industry concerns relating to the practical and theoretical basis for a risk-based planning framework informed by stochastic modelling assessments. 
Some of the issues may be resolved through improved communication between the academic and practitioner communities. For example, the view that synthetic drought scenarios generated by stochastic models are implausible might be refuted by academics in this field. Those promoting the use of stochastic generators may therefore need to somehow clearly explain why their models produce credible information for use in water resources planning. The view that industry lacks the necessary software, data and models to undertake stochastic water resources modelling assessments could be challenged using industry studies reported in the academic literature (e.g., Harris et al., 2013; Asefa et al., 2014; Borgomeo et al., 2014; Turner et al., 2014) or through modelling trials conducted as part of upcoming UKWIR research. Further method development and case demonstration will be required to give practitioners greater confidence that a risk-based planning framework can provide information that is useful for making and justifying investment decisions.

\section{Conclusions}

1. The study found that most practitioners acknowledge a number of weaknesses in DO assessment methodology and believe there is a case for more detailed examination of risk in the planning process. However, few see a strong case for a fundamental shift toward a risk-based planning approach informed by stochastic modelling assessments.

2. The study exposed a number of business risks associated with shifting away from conventional planning methods. Most importantly, several participants indicated that modelling assessments play an important role in building a consensus for action, which is more easily achieved when the state of a WRZ is presented deterministically using the supply-demand balance.

3. The study found that many practitioners are sceptical about the practicality and utility of risk-based methods in water resources planning. Some hold reservations about the industry's capacity for undertaking stochastic modelling assessments. Others distrust the outputs that emerge from such analyses or envisage difficulties in using the outputs to inform investment decisions.

\section{Acknowledgements}

This work was financially supported by the UK Engineering and Physical Sciences Research Council, United Utilities PLC, Anglian Water and Severn Trent Water through the STREAM Industrial Doctorate Centre. We thank all interview participants for participating in the study. Any opinions, findings, and conclusions or recommendations expressed in this paper (excluding the interview responses) are those of the authors and do not reflect the views of any of the organisations mentioned above. 


\section{References}

Asefa, T., Clayton, J., Adams, A., Anderson, D. (2014) Performance evaluation of water resources system under varying climate conditions: Reliability, resilience, vulnerability and beyond, Journal of Hydrology, 508, 53-65. doi: 10.1016/j.jhydrol.2013.10.043.

Borgomeo, E., J. W. Hall, F. Fung, G. Watts, K. Colquhoun, and C. Lambert (2014), Riskbased water resources planning: Incorporating probabilistic nonstationary climate uncertainties, Water Resour. Res., 50, 6850-6873, doi:10.1002/2014WR015558.Environment Agency, Ofwat, Defra and the Welsh Government (2012) Water Resources Planning Guideline - the technical methods and instructions.

Environment Agency (2013) Water Resources Management Plans 2019 - Preparing for the Future (Main Report), CH2M Hill, Swindon, 2013.

Davies, A. and Daykin, S. (2011) Review of Water Resources Management Planning process: final report, IHPR, Policy projects for CGL, DfT, DECC and Defra, PB13653.

DoE (1996) Water Resources and Supply: Agenda for Action, HMSO.

Foddy, W. (1993) Constructing questions for interviews and questionnaires - theory and practice in social research, Cambridge University Press, Cambridge.

Hall, J.W., Watts, G., Keil, M., de Vial, L., Street, R., Conlan, K., O’Connell, P.E., Beven, K.J., Kilsby, C.G. (2012) Towards risk-based water resources planning in England and Wales under a changing climate, Water and environment journal, 26, 118-129, doi: 10.1111/j.17476593.2011.00271.x.

Hall, J.W. and Borgomeo, E. (2013) Risk-based principles for defining and managing water security, Phil. Trans. R. Soc. A., 371:20120407, doi: 10.1098/rsta.2012.0407.

Harris, C.N.P., Quinn, A.D., Bridgeman, J. (2013) Quantification of uncertainty sources in a probabilistic climate change assessment of future water shortages, Climatic Change, 121(2), 317-329, doi: 10.1007/s10584-013-0871-8.

Korteling, B., Dessai, S., Kapelan, Z. (2013) Using information-gap decision theory for water resources planning under severe uncertainty, Water Resources Management, 27, 1149-1172, doi: 10.1007/s11269-012-0164-4.

Loucks, D.P., van Beek, E., Stedinger, J.R., Dijkman, J.P.M. and Villars, M.T. (2005) Water resources systems planning and management - an introduction to methods, models and applications. Unesco publishing.

Matrosov, E.S., Padula, S., Harou, J. (2013) Selecting portfolios of water supply and demand management strategies under uncertainty - contrasting economic optimisation and robust decision making approaches, Water Resources Management, 27, 1123-1148, doi: 10.1007/s11269-012-0118-x. 
S.W.D. Turner, R.J. Blackwell, M.A. Smith \& P.J. Jeffrey (2014): Risk-based water resources planning in England and Wales: challenges in execution and implementation, Urban Water Journal, doi: 10.1080/1573062X.2014.955856.

UK Infrastructure Transition Research Consortium (2014) National Infrastructure Assessment: Analysis of Options for Infrastructure Provision in Great Britain-Interim results, January 2014, Oxford. 